\title{
COMPLETIONS AND FIBRATIONS FOR TOPOLOGICAL MONOIDS
}

\author{
PAULO LIMA-FILHO
}

\begin{abstract}
We show that, for a certain class of topological monoids, there is a homotopy equivalence between the homotopy theoretic group completion $\mathrm{M}^{+}$ of a monoid $M$ in that class and the topologized Grothendieck group $\widetilde{M}$ associated to $M$. The class under study is broad enough to include the Chow monoids effective cycles associated to a projective algebraic variety and also the infinite symmetric products of finite $\mathrm{CW}$-complexes. We associate principal fibrations to the completions of pairs of monoids, showing the existence of long exact sequences for the naïve approach to Lawson homology [Fri91, LF91a]. Another proof of the Eilenberg-Steenrod axioms for the functors $X \mapsto \widetilde{S P}(X)$ in the category of finite CW-complexes (Dold-Thom theorem [DT56]) is obtained.
\end{abstract}

\section{INTRODUCTION}

The main goal of this article is to establish that for a certain class of topological monoids there is a natural homotopy equivalence between a suitably topologized Grothendieck group $\widetilde{M}$ associated to a monoid $M$ and the homotopy theoretic group-completion $M^{+} \stackrel{\text { def }}{=} \Omega B M$ obtained via classifying space theory. We also show the existence of principal fibrations for the Grothendieck group completions of pairs in the same category, which certainly makes this completion a very convenient functor.

The Grothendieck group $\widetilde{M}$ associated to a monoid $M$, usually denoted $\mathscr{K}(M)$, is the first and natural approach to the problem of finding group completions and it clearly carries the expected universal property when applied to discrete monoids, cf. $\S 2$. However, for topological categories, the problem poses many technical difficulties not previously encountered. One expects the completion to be sufficiently well behaved so as to have amenable algebraic properties, where reasonable manipulations and computations may be performed.

In the category of spaces, one could first try to use a naturally topologized Grothendieck group. However this turns out to be generically useless, for several reasons. Among the latter is the fact that the topologies arising in this way usually do not have the Hausdorff property and the Grothendieck group barely resembles a topological group.

Received by the editors April 8, 1991.

1991 Mathematics Subject Classification. Primary 55R35, 55P10; Secondary 55R05, 14C05, $55 \mathrm{~S} 15$.

Research partially supported by NSF Grant No. DMS- 8610730 through the Institute for Advanced Study. 
In the homotopy category one can circumvent the aforementioned difficulties with the use of classifying spaces for topological monoids. This is actually achieved in a general categorical framework through "Quillenization" type processes. Such homotopy theoretic description of the Grothendieck functor for topological categories can be found, for example, in [Seg74]. Direct applications for this approach are found in $\mathscr{K}$-theory [Qui73] and $\infty$-loop spaces theories [May72, Seg74]. When discussing classifying spaces for topological monoids, we use the topological bar construction, introduced in [Mil67], as described in greater generality in [May75]. In $\S 2$ we present a discussion of the basic properties of both completions.

There are some situations, however, where the more naïve Grothendieck group approach would be desirable for the sake of geometric clarity and intuition. Recently a certain interest on this point of view was raised with the advent of Lawson homology for projective varieties, introduced by E. Friedlander in [Fri91]. Those are invariants attached to a projective algebraic variety $X$ defined (in the complex case) as the homotopy groups of the group completion of the Chow monoid $\mathscr{C}_{p}(X)$ of effective $p$-cycles supported on $X$. See Example $B$ in $\S 4$. In the level of $\pi_{0}$ Lawson homology coincides with the group of algebraic cycles modulo algebraic equivalence, which turns out to be the Grothendieck group of $\pi_{0}\left(\mathscr{C}_{p}(X)\right)$, as shown in [Fri91]. Those are fundamental invariants for algebraic varieties, which are coarser than the usual Chow group of cycles modulo rational equivalence, but nonetheless carry a rich and beautiful geometry, as it can be seen for example in [Ful84, Chapter 19].

In this context one would like to retain the geometry of the space of cycles, as in the level of $\pi_{0}$, to the higher homotopy groups and this is directly achieved by the naive completion. Concomitantly one would hope the same algebraic properties encountered in the homotopy group completions to still exist. Surprisingly, this can be accomplished within a certain class (which we call properly $c$-graded monoids) consisting of those free abelian monoids whose topology is given by a compact grading with adequate cofibrant properties, as described in $\S 4$. More precisely, one has

Theorem 4.4. Let $M$ be a free properly c-graded topological monoid. Then the natural map $T: M^{+} \rightarrow \widetilde{M}$, between the homotopy and naïve group completions of $M$, is a homology equivalence. If $M$ is strongly properly c-graded, then $T$ is a quasifibration with contractible fibers, and hence a homotopy equivalence.

Corollary 4.5. The inclusion $j: M \hookrightarrow \widetilde{M}$ is a homology group completion for $M$ as in the theorem.

This class of monoids encompasses the Chow monoid of projective algebraic varieties as well as a number of other significant examples, such as the monoids $S P(X)$ arising as the (disjoint) union of the symmetric products of a finite $\mathrm{CW}$ complex, cf. $\S 3$. These examples will be discussed in detail along the paper, and some results of Dold-Thom [DT56] will follow from our general discussion.

In the last section we associate principal fibrations to the naive completions of free pairs of monoids as above, and relate them to the usual quasifibrations obtained via classifying space theory, cf. [May75]. We prove

Theorem 5.2. Given a properly c-filtered free pair of monoids $\left(C, C^{\prime}\right)$, there is a principal fibration $\widetilde{C}^{\prime} \rightarrow \widetilde{C} \rightarrow \widetilde{C} / \widetilde{C^{\prime}}$, where $\widetilde{C} / \widetilde{C^{\prime}}$ is the topological group quotient of the naïve group completions of $C$ and $C^{\prime}$, respectively. 
In particular one has long exact sequences for the Lawson homology of pairs, as defined in [LF89], and similar property for the corresponding functors $X \mapsto$ $\pi_{k}(\widetilde{S P}(X))$ on the category $\mathscr{W}_{0}$ of finite $\mathrm{CW}$-complexes.

The results of this paper form a simplified version of the author's unpublished Preprint Group completions and fibrations for filtered monoids, IAS, 1990.

The author has learned that, recently, E. Friedlander and O. Gabber have obtained a series of results which overlap with some of the results contained in the present work.

Acknowledgments. The author would like to thank Blaine Lawson for having first realized the importance of the equivalence of completions in the algebraic cycles situation; for his encouragement, optimism and firm belief in the truth of the result; and for having introduced the author to the geometry of the cycle space. A special debt of gratitude is due to E. Friedlander for having shared some of his early ideas on the equivalence of completions with the author, pointed to some technical difficulties and introduced the author to the homotopy of the cycle spaces. Finally the author thanks the hospitality of the Institute for Advanced Study during the elaboration of the main part of this work, and the referee for valuable suggestions.

\section{Preliminaries}

2.1. Naĩve group completions. In this section we work mainly with the categories of abelian monoids and abelian topological monoids.

Given an abelian monoid $M$, with identity element 0 , one can naturally associate a group $\widetilde{M}$ to $M$, together with a monoid morphism $j: M \rightarrow \widetilde{M}$ which has the universal property of factoring morphisms from $M$ into groups, i.e., given a monoid morphism $f: M \rightarrow G$ from $M$ into a group $G$, there exists a group homomorphism $\tilde{f}: \widetilde{M} \rightarrow G$ making the diagram commute:

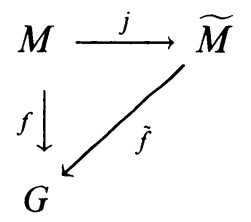

This universal group $\widetilde{M}$ can be obtained as the quotient of $M \times M$ by the equivalence relation $R$ defined as follows:

$$
\begin{gathered}
(x, y) \stackrel{R}{\sim}\left(x^{\prime}, y^{\prime}\right) \text { iff there exist } \lambda^{\prime}, \lambda \in M \text { such that } \\
(x+\lambda, y+\lambda)=\left(x+\lambda^{\prime}, y+\lambda^{\prime}\right),
\end{gathered}
$$

where + denotes the monoid operation. The morphism $j: M \rightarrow \widetilde{M}$ is then induced by the map $m \mapsto(m, 0)$ from $M$ into $M \times M$. Observe that $j$ is an injection whenever $M$ has cancellation law.

Remark 2.1. When cancellation law holds for $M$ the relation $R$ can be alternatively defined as follows. Let $\Gamma$ be the product $M \times M \times M \times M$ and define 


$$
\begin{aligned}
\Psi: \Gamma & \rightarrow M \times M, \\
\left(x, y ; x^{\prime}, y^{\prime}\right) & \mapsto\left(x+y^{\prime} ; x^{\prime}+y\right) .
\end{aligned}
$$

The relation $R \subset \Gamma$ is then given by $R=\Psi^{-1}(\Delta)$, where $\Delta \subset M \times M$ is the diagonal submonoid. The saturation (under $R$ ) of any subset $F \subset M \times M$ is obtained as

$$
\operatorname{Sat}_{R}(F)=\pi_{2}\left(\pi_{1}^{-1}(F) \cap \Psi^{-1}(\Delta)\right),
$$

where $\pi_{i}: \Gamma \rightarrow M \times M, i=1,2$, is the projection onto the $i$ th $M \times M$ factor of $\Gamma$.

Denoting by $\mathscr{A} \mathscr{M}$ (resp. $\mathscr{A} \mathscr{G}$ ) the category of abelian monoids (resp. abelian groups), the assignment of $\widetilde{M}$ to $M$ defines a functor $\sim: \mathscr{A} \rightarrow \mathscr{A} \mathscr{G}$ with the above universal property, which is called the (naïve) group completion or the Grothendieck group functor.

Our central interest lies in those objects in $\mathscr{A} \mathscr{M}$ which are also spaces. In this case we provide the Grothendieck group $\widetilde{M}$ with the topology induced by the quotient map $p: M \times M \rightarrow \widetilde{M}$.

Remark 2.2. Although $\widetilde{M}$ is not necessarily a topological group, it factors continuous monoid morphisms $f: M \rightarrow G$, from $M$ into a topological group $G$, through a continuous map $f: \widetilde{M} \rightarrow G$ which is a homomorphism in the category $\mathscr{A} \mathscr{M}$. In the compactly generated category (CG), with products in that category too, it can be seen, using Theorems 4.4 and 2.6 of [Ste67], that whenever $\widetilde{M}$ is Hausdorff then it has the structure of a compactly generated abelian topological group. In this case, the universal property is satisfied in the categories of topological monoids and groups. Therefore (in CG), the Grothendieck group becomes a functor $\sim: \mathscr{A} \mathscr{T} \mathscr{M} \rightarrow \mathscr{A T O G}$ from the category $\mathscr{A} \mathscr{G} \mathscr{M}$ of abelian topological monoids with Hausdorff naïve group completion, to the category $\mathscr{A} \mathscr{T} \mathscr{O} \mathscr{G}$ of abelian topological groups.

From now on our monoids will be compactly generated and products of spaces will be formed in that category, cf. [Ste67]. We must say that the Hausdorff property for the group completion does not hold generically, however it occurs in many interesting situations, as we shall see afterwards.

Lemma 2.3. For a given $M \in \mathscr{A} \mathscr{T} \mathscr{O M}$, the monoid morphism $j_{*}: \pi_{0}(M) \rightarrow$ $\pi_{0}(\widetilde{M})$ induced by the universal map $j: M \rightarrow \widetilde{M}$ is a group completion for the discrete monoid $\pi_{0}(M)$.

Proof. Since $\pi_{0}(\widetilde{M})$ is a group, we know that $j: \pi_{0}(M) \rightarrow \pi_{0}(\widetilde{M})$ factors through $\widetilde{\pi_{0}}(M)$ and we have a diagram:

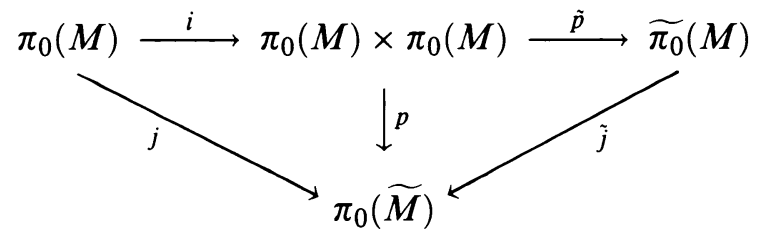

where $p$ is the map induced by the projection $p: M \times M \rightarrow \widetilde{M}$, and $\tilde{p}$ is the quotient map from $\pi_{0}(M) \times \pi_{0}(M)$ onto $\widetilde{\pi_{0}}(M)$. 
On the other hand, the composition of $\tilde{p} \circ i$ with the map $c: M \rightarrow \pi_{0}(M)$ sending an element of $M$ to its path component is a topological monoid morphism from $M$ into the discrete group $\widetilde{\pi_{0}}(M)$, and hence factors through $\widetilde{M}$ via a map $k: \widetilde{M} \rightarrow \widetilde{\pi_{0}}(M)$. It is now obvious that the induced map $\tilde{k}: \pi_{0}(\widetilde{M}) \rightarrow \widetilde{\pi_{0}}(M)$ is an inverse for $\tilde{j}: \widetilde{\pi_{0}}(M) \rightarrow \pi_{0}(\widetilde{M})$.

Definition 2.4. (i) For an object $\mathscr{A}$ in any category, we denote by $\mathscr{A}_{*}$ the simplicial object in the same category where each $\mathscr{A}_{p}$ is equal to $\mathscr{A}$ and the faces and degeneracies are the identity map.

(ii) We say that a topological monoid $M$ is $(c-)$ filtered if it has the weak topology given by a closed (compact) filtration $e=M_{0} \subseteq M_{1} \subseteq \cdots \subseteq M_{d} \subseteq \cdots$ by subspaces satisfying $M_{d}+M_{e} \subseteq M_{d+e}$.

(iii) A topological monoid $M$ is graded if there is a morphism of topological monoids deg: $M \rightarrow\left(\mathbb{Z}_{+}\right)$into the discrete monoid of nonnegative integers, such that $\operatorname{deg}^{-1}(0)=e=$ identity element of $M$. The monoid $M$ is $c$-graded if, in addition, each $M(n)=\operatorname{deg}^{-1}(n)$ is compact, for all $n \in \mathbb{Z}_{+}$.

(iv) A monoid $M$ is filtered by cofibrations if each inclusion $M_{d} \subseteq M_{d+1}$ is a cofibration. Recall that a map of spaces $f: A \rightarrow X$ is a cofibration if it is a monomorphism and has the homotopy extension property [Whi78]. The pair $(X, A)$ is often called an NDR-pair [Ste67].

Remark 2.5. Observe that a $(c-)$ graded abelian topological monoid $M$ is also (c-) filtered by the subspaces $M_{d}=\bigcup_{k \leq d} M(k)$. The product $M \times M$ of a (c-) filtered (resp. (c-) graded) topological monoid $M$ with itself becomes $(c-)$ filtered (resp. $(c-)$ graded) by $(M \times M)_{d} \stackrel{\text { def }}{=} \bigcup_{r+s \leq d} M_{r} \times M_{s}$ (resp. by $\operatorname{deg}(x, y)=\operatorname{deg} x+\operatorname{deg} y)$.

Remark 2.6. Recall that a map $f: X \rightarrow Y$ between topological spaces is called a proclusion [Ste67] whenever it is onto and $Y$ has the quotient topology induced by $f$. A map of pairs $f:(X, A) \rightarrow(Y, B)$ is a relative homeomorphism whenever $f: X \rightarrow Y$ is a proclusion and $f$ restricts to a homeomorphism $f: X-A \rightarrow Y-B$.

Lemma 2.7. Let $M$ be a c-filtered topological monoid with cancellation law. Then the subsets

$$
\widetilde{M}_{d} \stackrel{\text { def }}{=} p\left((M \times M)_{d}\right),
$$

are closed in $\widetilde{M}$ and the topology of $\widetilde{M}$ is the weak topology given by the filtration $\cdots \subseteq \widetilde{M}_{d-1} \subseteq \widetilde{M}_{d} \subseteq \widetilde{M}_{d+1} \subseteq \cdots$. Furthermore, the restriction of $p$ to $(M \times M)_{d}$ is a closed proclusion onto $\widetilde{M}_{d}$.

Proof. Since $M$ has cancellation law we know that

$$
\operatorname{Sat}_{R}(F)=\pi_{2}\left(\pi_{1}^{-1}(F) \cap \Psi^{-1}(\Delta)\right),
$$

for any $F \subseteq M \times M$, where $\pi_{i}: \Gamma \rightarrow M \times M, i=1,2$, is the projection onto the $i$ th $M \times M$ factor of $\Gamma=(M \times M) \times(M \times M)$, as shown in Remark 2.1. Recalling that a space $F$ is compact iff the projection $\mathrm{pr}_{2}: F \times X \rightarrow X$ is a closed map for all spaces $X$, one concludes from the above description that Sat $_{R}\left((M \times M)_{d}\right)$ is a closed subset of $M \times M$. In particular, $\widetilde{M}_{d}$ is a closed subset of $\widetilde{M}$. 
Suppose now that $S \subseteq \widetilde{M}$ satisfies $S \cap \widetilde{M}_{d}$ is closed in $\widetilde{M}_{d}$ for all $d$. Then $p^{-1}\left(S \cap \widetilde{M}_{d}\right)$ is closed in $M \times M$ and hence

$$
p^{-1}\left(S \cap \widetilde{M}_{d}\right) \cap(M \times M)_{d}=p^{-1}(S) \cap(M \times M)_{d}
$$

is closed for all $d$, showing that $S$ is closed in $\widetilde{M}$.

Since any closed subset $F$ of $(M \times M)_{d}$ is compact, its saturation is closed, as seen above. Therefore the restriction $\left.p\right|_{(M \times M)_{d}}$ is a closed surjection onto $\widetilde{M}_{d}$, and hence a closed proclusion.

2.2. Homotopy theoretic completions and quotients. Let us recall a functorial construction associated with topological monoids acting on spaces, "the triple bar construction," which has an important role in the theory of $\infty$-loop spaces [May72]. We use May's monograph [May75] as our standard reference.

Definition 2.8. Let $M$ be a topological monoid (with nondegenerate identity $e$ ) acting on spaces $X$ on the right and $Y$ on the left, with both actions denoted by *. Define $B_{*}(X, M, Y)$ as the simplicial space whose spaces of $k$-simplices $B_{k}$ is $X \times M^{k} \times Y$, and the faces $d_{i}$ and degeneracies $s_{i}$

$$
B_{k-1} \stackrel{d_{i}}{\longleftarrow} B_{k} \stackrel{s_{i}}{\longrightarrow} B_{k+1}, \quad i=0, \ldots, k,
$$

are defined as follows:

$$
\begin{aligned}
& d_{i}\left(x ; m_{1}, \ldots, m_{k} ; y\right) \\
& \quad= \begin{cases}\left(x * m_{1}, m_{2}, \ldots, m_{k} ; y\right), & i=0, \\
\left(x, m_{1}, \ldots, m_{i}+m_{i+1}, m_{i+2}, \ldots, m_{k} ; y\right), & 1 \leq i \leq k-1, \\
\left(x ; m_{1}, \ldots, m_{k+1} ; m_{k} * y\right), & i=k ;\end{cases} \\
& s_{i}\left(x ; m_{1}, \ldots, m_{k} ; y\right) \\
& = \begin{cases}\left(x ; e, m_{1}, \ldots, m_{k} ; y\right), & i=0, \\
\left(x, m_{1}, \ldots, m_{i}, e, m_{i+1}, \ldots, m_{k} ; y\right), & 1 \leq i \leq k-1, \\
\left(x ; m_{1}, \ldots, m_{k}, e ; y\right), & i=k .\end{cases}
\end{aligned}
$$

This is a functorial construction on triples $(X, M, Y)$ as above, in the sense that equivariant maps of triples induce morphisms between the corresponding simplicial spaces. We denote $B(X, M, Y)$ the geometric realization of $B_{*}(X, M, Y)$.

Remark 2.9. (a) Recall that the geometric realization $\left|B_{*}\right|$ of a simplicial space $B_{*}$ with faces and degeneracies given respectively by $d_{i}: B_{*} \rightarrow B_{*-1}$ and $s_{i}$ : $B_{*-1} \rightarrow B_{*}$, is defined as follows (see [May72, Mil57]): Let $\bar{B}_{*} \stackrel{\text { def }}{=} \coprod_{q \geq 0} B_{q} \times \Delta_{q}$, where

$$
\Delta_{q}=\left\{\left(t_{0}, \ldots, t_{q}\right) \mid 0 \leq t_{1} \leq 1, \sum t_{i}=1\right\} \subset \mathbb{R}^{q+1}
$$

is the standard topological $q$-simplex and $B_{q} \times \Delta_{q}$ has the product topology. Let $\delta_{i}: \Delta_{q-1} \rightarrow \Delta_{q}$ and $\sigma_{i}: \Delta_{q+1} \rightarrow \Delta_{q}$ be the standard geometric faces and degeneracies and define an equivalence relation $\sim$ on $B_{*}$ by

$$
\begin{array}{rlrl}
\left(d_{i} x, u\right) & \sim\left(x, \delta_{i} u\right), & & x \in B_{q}, u \in \Delta_{q-1}, \\
\left(s_{i} x, u\right) & \sim\left(x, \sigma_{i} u\right), & x \in B_{q}, u \in \Delta_{q+1}
\end{array}
$$


Set-theoretically we define $\left|B_{*}\right|$ to be $\bar{B}_{*} / \sim$. Now filter $\left|B_{*}\right|$ by the subsets $F^{p}\left|B_{*}\right|=\pi\left(\coprod_{i=0}^{p} B_{i} \times \Delta_{i}\right)$ where $\pi$ is the quotient map $\pi: \bar{B}_{*} \rightarrow\left|B_{*}\right|$ and give $F^{p}\left|B_{*}\right|$ the induced topology. Then $F^{p}\left|B_{*}\right|$ is closed in $F^{p+1}\left|B_{*}\right|$ and $\left|B_{*}\right|$ is given the direct limit topology of the $F^{p}\left|B_{*}\right|$ 's.

A point in $F^{p}\left|B_{*}\right|$ is usually denoted $\left|m_{p} ; t_{0}, u_{p}\right|$, where $m_{p} \in B_{p}$ and $u_{p}=\left(t_{0}, \ldots, t_{p}\right) \in \Delta_{p}$.

(b) It is easy to see that the property of being proclusive (Remark 2.6), commutes with direct limits and is preserved under composition. In particular, one sees that a geometric realization of a simplicial proclusion is again a proclusion. Also, if $X_{*}^{\prime}$ is a simplicial subspace of $X_{*}$ such that $X_{*}^{\prime} \hookrightarrow X_{*}$ is a cofibration cf. [May72], then the geometric realization $X^{\prime} \hookrightarrow X$ is again a cofibration.

A special case of a triple as above, is the one of a submonoid $N \subset M$ acting on $M$ by right translations and (trivially) on a point $x$. In this case, the space $B(M, N, x)$ is called the homotopy quotient of $M$ by $N$ and is denoted $B(M, N)$. Observe that whenever $M$ is abelian the quotient $B(M, N)$ is also a topological monoid, for $B_{*}(M, N, x)$ remains a simplicial topological monoid, cf. [May75].

We list below some properties and related examples to this particular case.

a. Define, for a topological monoid $M$, the spaces $E M \stackrel{\text { def }}{=} B(M, M, x)$ and $B M \stackrel{\text { def }}{=} B(x, M, x)$, where $M$ acts trivially on a point $x$. It is seen that for any $M$ acting on a space $Y$, there is a strong deformation retraction from $B(M, M, Y)$ onto $B(e, e, Y) \equiv Y$. In particular $E M$ is contractible.

b. In case $N \leq M$ is a group like submonoid, the sequence of maps $M \rightarrow B(M, N) \rightarrow B N$ induced by the natural maps of triples $(M, x, x) \rightarrow$ $(M, N, x) \rightarrow(x, N, x)$ is a principal quasifibration with structure monoid $M$ in the sense of [DL59]. When $M=N$, the sequence $M \rightarrow E M \rightarrow B M$ is the universal principal quasifibration for the monoid $M$, and $B M$ is the classifying space of $M$. For more details see [DL59, May75, Mil67]. In case $M$ is actually a topological group, the above construction provides a way of obtaining the classical classifying spaces for principal fibrations.

One has a natural map $u: M \rightarrow \Omega B M$, where $\Omega B M$ is the space of loops based on $|e ; 1| \in B M$, sending $m \in M$ to the loop $\gamma_{m}:[0,1] \rightarrow B M$ defined as $\gamma_{m}(t)=|m ; t, 1-t|$. See Remark 2.9 for the notation on points in the geometric realization of a simplicial space. This map has the property that the induced morphism $u_{*}: H_{*}(M) \rightarrow H_{*}(\Omega B M)$ between the Pontrjagin rings of $M$ and $\Omega B M$, respectively, is the localization of $H_{*}(M)$ with respect to the action of $\pi_{0}(M)$ on $H_{*}(M)$. For this reason, $M \rightarrow \Omega B M$ is called the homotopy theoretic or homology group completion of $M$. See [MS76] or [Qui] for details.

In the particular case in which $M$ is an abelian monoid, which encompasses all the cases we are interested in, one finds a better model for the homotopy theoretic group completion. Namely, consider the map

$$
u: M \rightarrow M^{+} \stackrel{\text { def }}{=} B(M \times M, M)
$$

induced by the natural map of triples $(M, x, x) \rightarrow(M \times M, M, x)$ sending $(m, x, x)$ to $(m \times 0,0, x)$, where $M$ acts on $M \times M$ as the diagonal submonoid. Then one has 
Proposition 2.10. For $M$ an abelian monoid, the map $u$ above is a homotopy theoretic group completion.

Proof. It follows from [May72, Lemma 11.11] that $u_{*}: \pi_{0}(M) \rightarrow \pi_{0}\left(M^{+}\right)$is exactly the group completion of the monoid $\pi_{0}(M)$ and hence that $M^{+}$is a group-like monoid. The universal property of the Pontrjagin ring $H_{*}(\Omega B M)$ implies that $u_{*}: H_{*} M \rightarrow H_{*} M^{+}$factors through $u_{*}: H_{*} M \rightarrow H_{*}(\Omega B M)$ via a ring homomorphism $T: H_{*}(\Omega B M) \rightarrow H_{*}\left(M^{+}\right)$.

We construct below a monoid morphism $\eta: M^{+} \rightarrow \Omega B M$ which gives the inverse of $T$ in homology. Observe that composition of loops in $\Omega B M$ is homotopy equivalent to the pointwise multiplication of loops induced by the abelian monoid structure in $B M$, and hence we use the latter as the operation in $\Omega B M$.

Let $X_{*}=S \wedge B_{*}(M \times M, M)$ be the simplicial space obtained as the reduced suspension of $B_{*}(M \times M, M)$, and whose geometric realization gives the reduced suspension of $M^{+}, \mathrm{cf}$. [May72, Proposition 12.1].

Define

$$
\begin{aligned}
& \bar{\eta}: \overline{X_{*}} \rightarrow \overline{B_{*} M}, \\
& {\left[\left(a, b ; m_{1}, \ldots, m_{q}\right) ; s\right], u_{q}} \\
& \mapsto \begin{cases}\left(a, m_{1}, \ldots, m_{q}\right),(1-2 s), 2 s u_{q} & \text { if } 0 \leq s \leq 1 / 2, \\
\left(b, m_{1}, \ldots, m_{q}\right),(2 s-1),(2-2 s) u_{q} & \text { if } 1 / 2 \leq s \leq 1,\end{cases}
\end{aligned}
$$

where $\overline{X_{*}}$ and $\overline{B_{*} M}$ are defined as in Remark 2.9, and $u_{q} \in \Delta_{q}$. It is an easy exercise to check that $\bar{\eta}$ induces a map in the level of geometric realizations $\eta: S M^{+} \rightarrow B M$ whose adjoint, still denoted $\eta: M^{+} \rightarrow \Omega B M$ is a morphism of topological monoids.

Notice that the following diagram of monoid morphisms commutes:

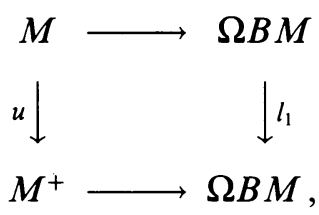

where, for $t \in[0,1], l_{t}: \Omega B M \rightarrow \Omega B M$ is the monoid morphism which sends $\gamma$ to $\gamma_{t}$ defined as

$$
\gamma_{t}(s)= \begin{cases}\gamma((1+t) s), & 0 \leq s \leq 1 /(1+t), \\ 0, & 1 /(1+t) \leq s \leq 1 .\end{cases}
$$

Using the universal property of $H_{*} M \rightarrow H_{*}(\Omega B M)$ and the above diagram one shows easily that $\eta_{*}$ is an inverse of the homomorphism $T$ above.

Remark 2.11. (a) The above proposition, whose proof was included as a referee's suggestion, is well known, although the elementary proof provided here is not found in the literature. In [Seg74, p. 305], Segal considers the simplicial space derived from the topological category $\mathscr{C}_{M}$ whose space of objects is $M \times M$, and whose space of morphisms from $\left(m_{1}, m_{2}\right)$ to $\left(m_{1}^{\prime}, m_{2}^{\prime}\right)$ is $\left\{m \in M: m_{1}+m=m_{1}^{\prime}, m_{2}^{\prime}+m=m_{2}^{\prime}\right\}$. This simplicial space is nothing else than $B_{*}(M \times M, M, x)$ and there he comments the fact that whenever $M$ is sufficiently homotopy commutative, then the construction provides a homotopy group completion for $M$. 
(b) This is a very amenable simplicial model for the homotopy group completion of an abelian monoid $M$ and will, henceforth, be denoted by $M^{+}$. When working with algebraic cycles, as in Example D of the next section, this construction will provide a simplicial algebraic model for the completion of the Chow monoid of an algebraic variety. This will prove to be very convenient.

Remark 2.12. Given an $\mathscr{A} \mathscr{T} \mathscr{O M} M$, the natural sequence of maps of triples $(M, x, x) \rightarrow(M \times M, M, x) \rightarrow(\widetilde{M}, x, x)$ is equivariant in the sense described in Definition 2.8, and hence it induces a commutative triangle of monoid morphisms:

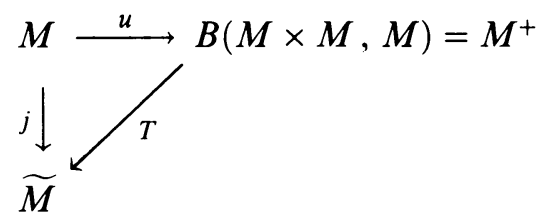

In particular, any morphism of topological monoids $f: M \rightarrow G$, from $M$ into a topological group $G$ factors through $M^{+}$.

\section{BASIC CONSTRUCTIONS AND EXAMPLES}

Given morphisms $f: M_{1} \rightarrow N$ and $g: M_{2} \rightarrow N$ of abelian topological monoids the map $f+g: M_{1} \times M_{2} \rightarrow N$ sending $\left(m_{1}, m_{2}\right)$ to $f\left(m_{1}\right)+$ $g\left(m_{2}\right)$ is again a morphism of topological monoids with the product structure. Throughout this paper we use the following notation: for a given morphism $f:\left(M, M^{\prime}\right) \rightarrow\left(N, N^{\prime}\right)$ of pairs of abelian topological monoids, denote by ${ }^{f} \psi$ the map

$$
{ }^{f} \psi: M \times N^{\prime} \rightarrow N
$$

defined by $f+i$, where $i: N^{\prime} \hookrightarrow N$ is the inclusion morphism.

One can naturally extend ${ }^{f} \psi$ to a simplicial map

$$
{ }^{f} \Psi_{*}: M \times E_{*} N^{\prime} \rightarrow B_{*}\left(N, N^{\prime}\right),
$$

and denote by

$$
f \Psi: M \times E N^{\prime} \rightarrow B\left(N, N^{\prime}\right),
$$

its geometric realization.

Our aim is to study situations where the "filtration" induced by ${ }^{f} \psi$ on its image behaves properly.

Definition 3.1. (a) Let $f:\left(M, M^{\prime}\right) \rightarrow\left(N, N^{\prime}\right)$ be a morphism of pairs of topological monoids, with $M$ a filtered monoid as in Definition 2.4. Define

$$
{ }^{f} B_{*}\left(N, N^{\prime}\right)_{d}={ }^{f} \Psi_{*}\left(M_{d} \times E_{*} N^{\prime}\right),
$$

and notice that ${ }^{f} B_{*}\left(N, N^{\prime}\right)_{d}$ is a simplicial subspace of $B_{*}\left(N, N^{\prime}\right)$. Similarly, define

$$
{ }^{f} B\left(N, N^{\prime}\right)_{d}={ }^{f} \Psi\left(M_{d} \times E N^{\prime}\right),
$$

which is simply the geometric realization of ${ }^{f} B_{*}\left(N, N^{\prime}\right)_{d}$ inside $B\left(N, N^{\prime}\right)$.

(b) Given $f$ as above, with $M$ a filtered monoid, define ${ }^{{ }^{f}} M_{d} \subseteq M_{d}$ as

$$
\begin{aligned}
{ }^{f} M_{d} & =\left\{m \in M_{d} \mid f(m)+n^{\prime}=f\left(m_{1}\right)+n^{\prime \prime} \text { for some } m_{1} \in M_{d-1}, n^{\prime}, n^{\prime \prime} \in N\right\} \\
& =\operatorname{pr}_{1}\left({ }^{f} \psi^{-1}\left({ }^{f} \psi\left(M_{d-1} \times N^{\prime}\right)\right) \cap\left(M_{d} \times N^{\prime}\right)\right),
\end{aligned}
$$


where $\mathrm{pr}_{1}$ is the projection from $M \times N^{\prime}$ onto $M$. Notice the interpolation $M_{d-1} \subseteq{ }^{f} M_{d} \subseteq M_{d}$.

Lemma 3.2. The restriction of ${ }^{f} \Psi$ to $\left(M_{d} \backslash^{f} M_{d}\right) \times E N^{\prime}$ maps one-to-one and onto ${ }^{f} B\left(N, N^{\prime}\right)_{d} \backslash^{f} B\left(N, N^{\prime}\right)_{d-1}$.

Proof. Exercise in geometric realizations.

Our first two examples below are of fundamental importance to the rest of the paper. They carry the key technical ingredients to prove our results.

Example A. Let $C$ be a given filtered monoid. Consider the pair $(M, N)$, where $M=C \times C$ with the product filtration of the previous section, and $N=\Delta \cong C$ is the diagonal submonoid. Let $f=$ id : $(C \times C, C) \rightarrow(C \times C, C)$ be the identity map. Notice that id $(C \times C)_{d}$ consists of those elements $\left(c, c^{\prime}\right) \in$ $(C \times C)_{d}$ which are equivalent to some element in $(C \times C)_{d-1}$ under the equivalence relation defining the Grothendieck completion $\widetilde{C}$ of $C$. Here $(C \times C)_{d}$ is the filtration in $C \times C$ induced by that of $C$, cf. Remark 2.5.

In this case ${ }^{\text {id }} \psi:(C \times C) \times C \rightarrow C \times C$ is surjective and proclusive, for if ${ }^{\text {id }} \psi^{-1}(F)$ is closed in $(C \times C) \times C$ for some subset $F \subseteq C \times C$, then $F={ }^{\text {id }} \psi^{-1}(F) \cap(C \times C \times 0)$, which is certainly closed in $C \times C=(C \times C) \times 0$. Here 0 denotes the unity element of the monoid. It follows from Theorem 4.4

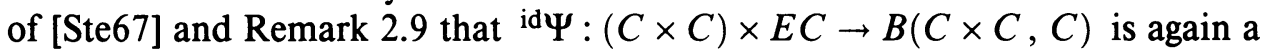
surjective proclusion.

Example B. Let $C$ be $c$-graded (compactly graded), and let $C^{\prime}$ be a closed submonoid of $C$. Then $\left(C^{\prime} \times C^{\prime}\right)+\Delta$ is a closed submonoid of $C$, which we denote $\Delta_{C^{\prime}}$. The latter assertion follows from the fact that $\left(\left(C^{\prime} \times C^{\prime}\right)+\Delta\right) \cap$ $(C \times C)_{d}$ is equal to

$$
\left(\bigcup_{a+2 b \leq d}\left(C^{\prime} \times C^{\prime}\right)_{a}+\Delta_{b}\right) \cap(C \times C)_{d}
$$

by the grading hypothesis, and from the compactness of $\left(\bigcup_{a+2 b \leq d}\left(C^{\prime} \times C^{\prime}\right)_{a}+\right.$ $\left.\Delta_{b}\right)$. A similar reasoning shows that the map

$$
{ }^{\text {id }} \psi:(C \times C) \times \Delta_{C^{\prime}} \rightarrow(C \times C)
$$

is a closed surjection, and hence it is proclusive. Again, it follows from [Ste67, Theorem 4.4] and Remark 2.9, that the map

$$
\text { id } \Psi:(C \times C) \times E\left(\Delta_{C^{\prime}}\right) \rightarrow B\left(C \times C, \Delta_{C^{\prime}}\right)
$$

is a closed proclusion. In particular, the $c$-grading assumption in conjunction with Theorem 9.5 of [Ste67] (see first paragraph of Step 3 in the proof of Proposition 4.2) imply that the topology of $M^{+} \stackrel{\text { def }}{=} B(C \times C, C)$ is given by the filtration

$$
{ }^{\text {id }} B(C \times C, C)_{d-1} \subseteq{ }^{\text {id }} B(C \times C, C)_{d} \subseteq{ }^{\text {id }} B(C \times C, C)_{d+1} \cdots .
$$

The following two examples are to be seen as particular instances of the two previous ones. 
Example C (Symmetric products). Let $X$ be a finite, connected (for convenience) $\mathrm{CW}$-complex, and let

$$
S P_{d}(X) \stackrel{\text { def }}{=}(X \times \cdots \times X) / \Sigma_{d},
$$

where $\Sigma_{d}$ is the symmetric group on $d$ letters acting by permuting the coordinates. Endowed with the quotient topology $S P_{d}(X)$ is the $d$-fold symmetric product of $X$, and, after a suitable subdivision of the product cell structure on $X \times \cdots \times X$, it can be given a quotient $\mathrm{CW}$-complex structure.

Let $S P_{0}(X)$ be an isolated point $\underline{0}$ not in $X$, and define

$$
S P(X) \stackrel{\text { def }}{=} \coprod_{d \geq 0} S P_{d}(X)
$$

taken with the disjoint union topology. It is easily seen that $S P(X)$ has the structure of a free $c$-graded abelian topological monoid, where $\underline{0}$ is the unity and the multiplication is given (additive notation) by

$$
\begin{gathered}
S P_{d}(X) \times S P_{e}(X) \rightarrow S P_{d+e}(X), \\
\left(\left\{x_{1}, \ldots, x_{d}\right\},\left\{y_{1}, \ldots, y_{e}\right\}\right) \mapsto\left\{x_{1}, \ldots, x_{d} ; y_{1}, \ldots, y_{e}\right\} .
\end{gathered}
$$

The choice of a fixed point $x_{0} \in X$ defines an embedding $S P_{d}(X) \stackrel{+x_{0}}{\hookrightarrow}$ $S P_{d+1}(X)$ and one defines [DT56] the classical infinite symmetric product of the space $X$ as the direct limit

$$
S P_{\infty}(X)=\underset{\lim }{\longrightarrow} S P_{d}(X)
$$

with respect to these inclusions.

The assignment of $S P(X)$ (resp. $\left.S P_{\infty}(X)\right)$ to $X$ is a functor from the category $\mathscr{W}_{0}$ (resp. $\mathscr{W}_{0^{*}}$ ) of finite $\mathrm{CW}$-complexes (resp. finite pointed $\mathrm{CW}$ complexes) to the category of topological monoids.

Remark 3.3. Note that, from Proposition 4.2 and subsequent remark, both $S P(X)$ and $S P_{\infty}(X)$ are $\mathscr{A} \mathscr{T} \mathscr{O M}$ 's, i.e., they have Hausdorff naïve completions, and also that their naïve group completions are filtered by cofibrations. In Dold-Thom's paper [DT56] the group $\widetilde{S P}(X)$ is denoted $A G(X)$.

There is a natural surjective morphism of $\mathscr{A} \mathscr{T} \mathscr{O M}$ 's $\pi: S P(X) \rightarrow S P_{\infty}(X)$ which induces a morphism of topological groups $\pi: \widetilde{S P}(X) \rightarrow \widetilde{S P_{\infty}}(X)$.

Lemma 3.4. The map $\pi$ above induces an isomorphism $\pi: \widetilde{S P}(X)_{0} \stackrel{\sim}{\rightarrow} \widetilde{S P_{\infty}}(X)$ between the connected component of the identity $\widetilde{S P}(X)_{0} \subset \widetilde{S P}(X)$ and $\widetilde{S P_{\infty}}(X)$. Proof. Immediate from the definitions.

Example D (Algebraic cycles). An (effective) algebraic p-cycle in the complex projective space $\mathbb{P}^{N}$ is a finite formal sum $\sigma=\sum_{\lambda} n_{\lambda} V_{\lambda}$, where the $n_{\lambda}$ 's are positive integers and the $V_{\lambda}$ 's are (irreducible) subvarieties of dimension $p$ in $\mathbb{P}^{N}$.

The degree of $\sigma=\sum_{\lambda} n_{\lambda} V_{\lambda}$ is defined as $\sum_{\lambda} n_{\lambda} \operatorname{deg}\left(V_{\lambda}\right)$, where $\operatorname{deg} V_{\lambda}$ is the degree of the irreducible subvariety $V_{\lambda}$ in $\mathbb{P}^{N}$, and the support of $\sigma$ is the algebraic subvariety $\bigcup_{\lambda} V_{\lambda}$ of $\mathbb{P}^{N}$.

The set of $p$-cycles of a fixed degree $d$ in $\mathbb{P}^{N}$ can be given the structure of an algebraic set, which we denote by $C_{p, d}\left(\mathbb{P}^{N}\right), \mathrm{cf}$. [Sam 55 and Sha74]. In case 
$X \subseteq \mathbb{P}^{N}$ is an algebraic subvariety of $\mathbb{P}^{N}$, the subset $C_{p, d}(X) \subset C_{p, d}\left(\mathbb{P}^{N}\right)$, consisting of those cycles whose support is contained in $X$, is an algebraic subvariety of $C_{p, d}\left(\mathbb{P}^{N}\right)$. See [Fri91, Hoy66, Sam55].

Definition 3.5. The set

$$
\mathscr{C}_{p}(X)=\coprod_{d \geq 0} C_{p, d}(X)=\{\underline{0}\} \amalg\left\{\coprod_{d>0} C_{p, d}(X)\right\}
$$

of all effective $p$-cycles in $X$, together with an isolated point $\underline{0}$ ("zero cycle") is an abelian topological monoid under cycle addition, called the Chow monoid of effective $p$-cycles on $X$. Here we are considering the analytic topology on $\mathscr{C}_{p}(X)$.

Given a closed algebraic subvariety $X^{\prime} \subseteq X$, the Chow monoid $\mathscr{C}_{p}\left(X^{\prime}\right)$ is naturally a closed (algebraic) submonoid of $\mathscr{C}_{p}(X)$. In particular the inclusions $\mathscr{C}_{p, d}\left(X^{\prime}\right) \subseteq \mathscr{C}_{p, d}(X)$ are cofibrations. This can be seen using triangulations compatible with the inclusions as in [Hir75].

The Chow monoid $\mathscr{C}_{p}(X)$ is a $c$-graded, free topological monoid, whose addition is a semialgebraic map, cf. [Fri91], when restricted to the graded pieces. In particular, the action of the diagonal of $\mathscr{C}_{p}(X) \times \mathscr{C}_{p}(X)$ is semialgebraic and, using triangulations once again [Hir75], one concludes that $\mathscr{C}_{p}(X)$ is an $\mathscr{A} \mathscr{T} \mathscr{O M}$ and its naïve group completion is filtered by cofibrations, cf. Proposition 4.2.

\section{EQUiVALENCE OF GROUP COMPLETIONS}

In this section we establish the convention that, whenever $M$ is a filtered topological monoid, then, in accordance with Definition 3.1, the subset id $(M \times M)_{d}$ is defined with regard to the identity map id $:(M \times M, M) \rightarrow$ $(M \times M, M)$, where $M \cong \Delta \subset M \times M$ is the diagonal submonoid. See Example A for a detailed description of id $(M \times M)_{d}$.

Definition 4.1. We say that $M$ is properly filtered whenever its filtration $\cdots \subseteq$ $M_{d-1} \subseteq M_{d} \subseteq M_{d+1} \subseteq \cdots$ has the property that the inclusion ${ }^{\text {id }}(M \times M)_{d} \subseteq$ $(M \times M)_{d}$ is a cofibration. $M$ is properly graded is the filtration comes from a grading as in Remark 2.5, and $M$ is strongly properly filtered (or graded) if the pair $\left((M \times M)_{d},{ }^{\text {id }}(M \times M)_{d}\right)$ is a strong NDR-pair, see Appendix of [May72]. Whenever the filtration is by compact subsets the corresponding monoids are called properly $c$-filtered, properly $c$-graded, strongly properly $c$-filtered and strongly properly $c$-graded, accordingly.

Proposition 4.2. Let $M$ be a free properly c-graded topological monoid. Then $M$ is an $\mathscr{A} \mathscr{T O M}$ and $\widetilde{M}$ is filtered by cofibrations.

Proof. Step 1. First observe that the restriction

$$
p:(M \times M)_{d} \backslash^{\mathrm{id}}(M \times M)_{d} \rightarrow \widetilde{M}_{d} \backslash \widetilde{M}_{d-1}
$$

is bijective, for whenever $\left(m_{1}, m_{2}\right),\left(m_{1}^{\prime}, m_{2}^{\prime}\right) \in(M \times M)_{d} \backslash^{d}(M \times M)_{\text {id }}$ satisfy $p\left(m_{1}, m_{2}\right)=p\left(m_{1}^{\prime}, m_{2}^{\prime}\right)$ then

$$
m_{1}+m_{2}^{\prime}=m_{1}^{\prime}+m_{2}
$$

by cancellation law, according to Remark 2.1. We may assume that $m_{1}$ and $m_{2}$ as well as $m_{1}^{\prime}$ and $m_{2}^{\prime}$ have no common factors in their expressions as sums of 
generators. Otherwise, supposing that $\left(m_{1}, m_{2}\right)=\left(n_{1}+\lambda, n_{2}+\lambda\right)$ with $\lambda \neq 0$, one gets

$$
d \geq \operatorname{deg}\left(m_{1}\right)+\operatorname{deg}\left(m_{2}\right)=\operatorname{deg}\left(n_{1}\right)+\operatorname{deg}\left(n_{2}\right)+\operatorname{deg}(\lambda)>\operatorname{deg}\left(n_{1}\right)+\operatorname{deg}\left(n_{2}\right),
$$

which would imply that $\left(m_{1}, m_{2}\right) \in{ }^{\text {id }}(M \times M)_{d}$. Now, the freeness assumption together with identity (4.2.1) implies that $m_{1}=m_{1}^{\prime}$ and $m_{2}=m_{2}^{\prime}$.

Step 2. We prove that each $\widetilde{M}_{d}$ is Hausdorff using induction. Recall that $\widetilde{M}_{0}$ is a point and assume that $\widetilde{M}_{d-1}$ is Hausdorff for $d>1$.

Since the inclusion id $(M \times M)_{d} \subseteq(M \times M)_{d}$ is a cofibration, one has, in particular, a "separating" function $u:[0,1] \rightarrow(M \times M)_{d}$, satisfying $u^{-1}(0)=$ id $(M \times M)_{d}$ and a retraction $r: u^{-1}([0,1)) \rightarrow{ }^{\text {id }}(M \times M)_{d}$. Given two points $\alpha$ and $\beta$ in $\widetilde{M}_{d}$, there are three possibilities:

(i) $\alpha, \beta \in \widetilde{M}_{d-1}$. In this case, by induction, there are disjoint open subsets $\widehat{U}, \widehat{V} \subseteq \widetilde{M}_{d-1}$ containing $\alpha$ and $\beta$ respectively. The inverse images

$$
U=p^{-1}(\widehat{U}) \cap \text { id }(M \times M)_{d} \quad \text { and } \quad V=p^{-1}(\widehat{V}) \cap \text { id }(M \times M)_{d}
$$

are disjoint open subsets of id $(M \times M)_{d}$. It is now easy to see, using Step 1 that $r^{-1}(U)$ and $r^{-1}(V)$ are disjoint open subsets of $(M \times M)_{d}$ which satisfy $p^{-1}\left(p\left(r^{-1}(U)\right)\right)=r^{-1}(U)$ and $p^{-1}\left(p\left(r^{-1}(V)\right)\right)=r^{-1}(V)$. Therefore, $p\left(r^{-1}(U)\right)$ and $p\left(r^{-1}(V)\right)$ are disjoint open subsets of $\widetilde{M}_{d}$ (cf. Lemma 2.7) containing $\alpha$ and $\beta$, respectively.

(ii) $\alpha \in \widetilde{M}_{d-1}$ and $\beta \in \widetilde{M}_{d} \backslash \widetilde{M}_{d-1}$. This is equivalent to $u(\bar{\alpha})=0$ and $u(\bar{\beta}) \neq 0$, where $\bar{\alpha} \in{ }^{\text {id }}(M \times M)_{d}$ and $\bar{\beta} \in(M \times M)_{d} \backslash^{d}(M \times M)_{\text {id }}$ are preimages of $\alpha$ and $\beta$ respectively. Using Step 1 and Lemma 2.7 one sees, as before, that $p\left(u^{-1}([0, u(\beta) / 3))\right)$ and $p\left(u^{-1}(2 u(\beta) / 3,1)\right)$ are disjoint open subsets of $\widetilde{M}_{d}$ separating $\alpha$ and $\beta$.

(iii) $\alpha$ and $\beta \in \widetilde{M}_{d} \backslash \widetilde{M}_{d-1}$. Apply Lemma 2.7 to the projections under $p$ of two disjoint open subsets $U$ and $V$ which separate the preimages of $\alpha$ and $\beta$ in $(M \times M)_{d} \backslash^{d}(M \times M)_{\text {id }}$.

Step 3. Observe that the restriction of a proclusion to a closed subset is again proclusive onto its image. Also observe that a map of pairs $f:(X, A) \rightarrow$ $(Y, B)$, with $f: X \rightarrow Y$ a proclusion and $A$ and $B$ closed subsets, is a relative homeomorphism if and only if $f: X-A \rightarrow Y-B$ is one-to-one and onto.

Using the previous paragraph, together with Lemma 2.7, Step 1 and Step 2, one sees that

$$
p:\left((M \times M)_{d},{ }^{d}(M \times M)_{\mathrm{id}}\right) \rightarrow\left(\widetilde{M}_{d}, \widetilde{M}_{d-1}\right)
$$

is a relative homeomorphism and hence that the inclusion $\widetilde{M}_{d-1} \subseteq \widetilde{M}_{d}$ is a cofibration, according to [Ste67, Lemma 8.4]. Since $\widetilde{M}$ is filtered by the $\widetilde{M}_{d}$ 's, Theorem 9.4 of [Ste67] shows that $\widetilde{M}$ is Hausdorff.

Remark 4.3. The hypothesis $M$ free and graded are slightly restrictive in the above proposition. It can be weakened to include $c$-filtered monoids whose filtration satisfies the following freeness-type property: whenever $p(a, b)=$ $p\left(a^{\prime}, b^{\prime}\right)$ for $(a, b) \in(M \times M)_{d}$, then either $(a, b)=\left(a^{\prime}, b^{\prime}\right)$ or $(a, b)=$ id $(M \times M)_{d}$. For example $S P_{\infty}(X)$, for $X$ a finite CW-complex, satisfies this property. This freeness for the filtration suffices to ensure the validity of Step 
1 in the proof of the proposition. The other steps remain unchanged. See Example 4.6.

Our aim is to prove the following result.

Theorem 4.4. Let $M$ be a free properly c-graded topological monoid. Then the natural map $T: M^{+} \rightarrow \widetilde{M}$ is a homology equivalence.

Corollary 4.5. The inclusion $j: M \hookrightarrow \widetilde{M}$ is a homology group completion whenever $M$ is properly filtered.

Proof. Recall the diagram of Remark 2.12. The inclusion $j: M \rightarrow \widetilde{M}$ factors through $u: M \rightarrow M^{+}$as $j=T \circ u$, where $T: M^{+} \rightarrow \widetilde{M}$ is the homotopy equivalence of the theorem. Since $M$ is abelian, the maps $j, u$, and $T$ are monoid morphisms, and hence $T$ induces an isomorphism between Pontrjagin rings $T_{*}: H_{*}(B(M \times M, M)) \rightarrow H_{*}(\widetilde{M})$. Using Lemma 2.3 and Proposition 2.10 one concludes the proof.

If one requires the cofibrations in Definition 4.1 to be strong NDR-pairs (see Appendix of [May72] for definition) then a stronger statement holds:

Theorem 4.4'. Let $M$ be a free, strongly properly c-graded topological monoid. Then the natural map $T: M^{+} \rightarrow \widetilde{M}$ is a quasifibration with contractible fibers. In particular it is a homotopy equivalence.

Example 4.6. A. Dold-Thom type results $I$. Consider the free monoids $S P(X)$ and $S P_{\infty}(X)$ in Example $C$ of $\S 3$, for $X$ finite CW-complex, and recall that the first one is $c$-graded while the latter is $c$-filtered. Both monoids are properly $c$-filtered and this follows from successive "subdivisions" of $(S P(X) \times S P(X))_{d}$ compatible with id $(S P(X) \times S P(X))_{d}$, and similarly for $\left(S P_{\infty}(X) \times S P_{\infty}(X)\right)_{d}$.

As noted in Remark 4.3 one can show that $S P_{\infty}(X)$, although only $c$-filtered, still satisfies the conclusion of Theorem 4.4 .

It follows from Corollary 4.5 that $\widetilde{S P}(X)$ and $\widetilde{S P_{\infty}}(X)$ are models for the homology group completion of $S P(X)$ and $S P_{\infty}(X)$, respectively. Furthermore, since $S P_{\infty}(X)$ is connected, the inclusion $u: S P_{\infty}(X) \rightarrow \widetilde{S P_{\infty}}(X)$ is a homotopy equivalence, by the group completion theorem [MS76] and Corollary 4.5.

On the other hand one has $\pi_{0}(\widetilde{S P}(X)) \cong \pi_{0}(\widetilde{S P}(X)) \cong \mathbb{Z}$ which is isomorphic to $H_{0}(X, \mathbb{Z})$. The higher homotopy groups of $\widetilde{S P}(X)$ and $S P_{\infty}(X)$ are isomorphic by Lemma 3.4 and the previous paragraph.

B. Algebraic cycle spaces and Lawson homology I. The Chow monoids $\mathscr{C}_{p}(X)$ of a closed projective variety $X$ defined on Example D form another family of examples of free, strongly properly $c$-graded monoids. The latter property is again seen by using triangulation of algebraic sets (cf. [Hir75]) and the fact that the graded components of the monoid are algebraic sets and the monoid operation is semialgebraic.

The homotopy groups of the homology group-completion of $\mathscr{C}_{p}(X)^{+}$form a set of invariants for the algebraic set called the Lawson homology of $X$ and were introduced by Friedlander in [Fri91], following the work of Lawson [Law89]. They are shown to be highly interesting invariants which comprehend many classical invariants. For example, Friedlander shows that the Néron-Severi group $N S(X), H^{1}\left(\operatorname{Pic}^{0}(X)\right)$ and the classical Chow groups of algebraic cycles modulo 
algebraic equivalence are particular cases of Lawson homology. Recent developments of this theory can be found in [FL, FM91, LF91a, LF91b].

The above corollary shows that one can replace the homotopy theoretic groupcompletion by the naive one in the definition of Lawson homology (over the complex numbers), with the gain of having a better geometric intuition on our side.

Observe the existence of a commutative diagram

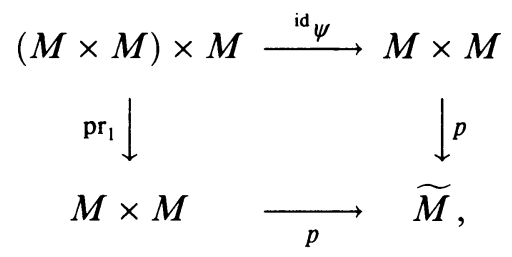

where $\mathrm{pr}_{1}$ is the projection on the two first factors and $p$ is the quotient map described in $\S 2$. Therefore, by functoriality of the bar construction, the following diagram commutes:

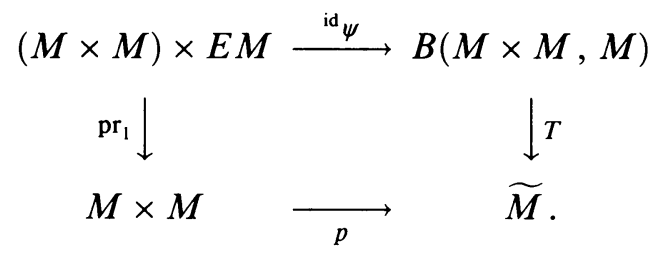

Proposition 4.7. Under the hypothesis of the theorem there is, for every $d \geq 1$, a commutative diagram of pairs

$$
\begin{aligned}
& \left((M \times M)_{d},{ }^{\mathrm{id}}(M \times M)_{d}\right) \times E M \stackrel{\mathrm{id} \Psi}{\longrightarrow}\left({ }^{\mathrm{id}} M_{d}^{+},{ }^{\mathrm{id}} M_{d-1}^{+}\right) \\
& \mathrm{pr}_{1} \downarrow \downarrow \downarrow T \\
& \left((M \times M)_{d},{ }^{\mathrm{id}}(M \times M)_{d}\right) \quad \underset{p}{\longrightarrow}\left(\widetilde{M}_{d}, \widetilde{M}_{d-1}\right),
\end{aligned}
$$

whose horizontal arrows are relative isomorphisms of cofibrations.

Proof. The bottom map was studied in Proposition 4.2. As to the top one, observe that, from the definition of properly filtered monoids and the fact that cofibrations are preserved under products, cf. [Ste67, Theorem 6.3], the upper left pair of the square is a cofibration. Once we show that ${ }^{\text {id }} \Psi$ is a relative homeomorphism, it follows, from [Ste67, Lemma 8.4], that the pair on the upper right corner is a cofibration.

We have seen in Lemma 3.2 that

$$
{ }^{f} \Psi:\left((M \times M)_{d}-{ }^{\mathrm{id}}(M \times M)_{d}\right) \times E M \rightarrow\left({ }^{\mathrm{id}} M_{d}^{+},{ }^{\mathrm{id}} M_{d-1}^{+}\right)
$$

is one-to-one and onto. Therefore it suffices to show that id $\psi:(M \times M) \times$ $E M \rightarrow M^{+}$is a closed proclusion to conclude that the top map is a relative isomorphism. See Step 3 in the proof of Proposition 4.2.

In Example B we have shown that id $\psi:(M \times M) \times M \rightarrow M \times M$ is a closed

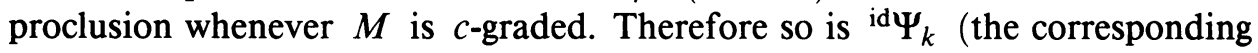


map on $k$-simplices), again by [Ste67, Theorem 6.3]. Since geometric realization preserves proclusions, cf. Remark 2.9, we have proven that the top map in the diagram is a relative isomorphism.

We now prove the main theorems.

Proof of Theorem 4.4. We use induction to show that $T:{ }^{\text {id }} M_{d}^{+} \rightarrow \widetilde{M}_{d}$ is a homology equivalence. For $d=0$ note that

$$
{ }^{\text {id }} M_{0}^{+} \stackrel{\text { def }}{=} \text { id } \Psi\left((M \times M)_{0} \times E M\right)=B(\Delta, \Delta) \cong E M,
$$

where $\Delta$ is the diagonal in $M \times M$. Since $E M$ is contractible, $T:{ }^{\text {id }} M_{0}^{+} \rightarrow$ $\widetilde{M}_{0}=\underline{\tilde{0}}$ is a homology equivalence.

Consider the diagram of Proposition 4.7 above, with $d \geq 1$. Using Proposition 4.7 and [Whi78, $\S 3$ ], one shows that both ${ }^{\text {id }} \Psi$ and $p$ induce isomorphisms between the relative homology of the pairs involved.

The contractibility of $E M$ and the commutativity of the diagram make $T$ induce an isomorphism in relative homology, too. By induction and the five lemma the theorem is proven once we observe that $M^{+}$is the direct limit of the filtration $\cdots \subseteq \subseteq{ }^{\text {id }} M_{d-1}^{+} \subseteq{ }^{\text {id }} M_{d}^{+} \subseteq{ }^{\text {id }} M_{d+1}^{+} \subseteq \cdots$, cf. Example B.

Proof of Theorem $4.4^{\prime}$. Let us say that $U \subseteq \widetilde{M}$ is distinguished if the restriction $T: T^{-1}(U) \rightarrow U$ is a quasifibraton.

(a) Observe that $T^{-1}\left(\widetilde{M}_{d}\right)={ }^{\text {id }} M_{d}^{+}$and that Proposition 4.7 yields a commutative diagram

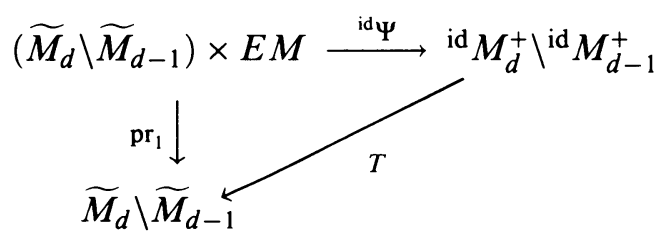

where the top horizontal arrow is a homeomorphism. This shows that any open subset $U \subset \widetilde{M}_{d} \backslash \widetilde{M}_{d-1}$ is distinguished, for all $d \geq 1$. The identity $\widetilde{M}_{0}$ is obviously distinguished.

(b) Let

$$
u:(M \times M)_{d} \rightarrow I, \quad h: I \times(M \times M)_{d} \rightarrow(M \times M)_{d}
$$

be a representation of the pair $\left((M \times M)_{d},(M \times M)_{d-1}\right)$ as a strong NDR-pair. Let $v=u \circ \mathrm{pr}_{1}$ and $H=h \circ\left(\mathrm{id} \times \mathrm{pr}_{1}\right) \times$ id be the NDR representation for the product of the previous pair with $E M$. Using [Ste67] and Proposition 4.7 we have corresponding representations $\tilde{u}, \tilde{h}$ and $\tilde{v}, \widetilde{H}$ for the pairs $\left(\widetilde{M}_{d}, \widetilde{M}_{d-1}\right)$ and $\left({ }^{\text {id }} M_{d}^{+}\right.$, id $\left.M_{d-1}^{+}\right)$, satisfying $\tilde{u} \circ p=u, \tilde{h} \circ(\operatorname{id} \times p)=h$ and $\tilde{v} \circ T=v$, $\widetilde{H} \circ(\operatorname{id} \times$ id $\Psi)=H$, respectively.

Let $\widetilde{U}=\tilde{u}^{-1}([0,1))$. The strong NDR condition implies that the homotopy $\tilde{h}$ restricts to $\tilde{h}_{y}: \widetilde{U} \rightarrow \widetilde{U}$ and by construction of the NDR representations above, the homotopy $\widetilde{H}_{t}: p^{-1}(\widetilde{U}) \rightarrow p^{-1}(\widetilde{U})$ covers the homotopy $\tilde{h}_{t}$, i.e., $T \circ \widetilde{H}_{t}=\tilde{h}_{t} \circ T$.

(c) At last, observe that the fiber $T^{-1}(\tilde{m})$ is isomorphic to $E M$ for all $\tilde{m} \in \widetilde{M}$, and hence $\widetilde{H}_{1}: T^{-1}(\tilde{m}) \rightarrow T^{-1}\left(h_{1}(\tilde{m})\right)$ is a homotopy equivalence for all $\tilde{m} \in \tilde{U}$. 
Conditions (a)-(c) imply that $T$ is a quasifibration, cf. Lemma 7.2 in [May72].

\section{FIBRATIONS FOR PROPERLY FILTERED PAIRS}

In this section we establish the convention that, whenever $\left(C, C^{\prime}\right)$ is a pair of filtered topological monoids, then ${ }^{i d}(C \times C)_{d}$ is defined with regard to the identity map

$$
\text { id : }\left(C \times C, \Delta_{C^{\prime}}\right) \rightarrow\left(C \times C, \Delta_{C^{\prime}}\right),
$$

where $\Delta_{C^{\prime}} \subseteq C \times C$ is the submonoid $\left(C^{\prime} \times C^{\prime}\right)+\Delta$ and $\Delta \cong C$ is the diagonal submonoid, as described in Example B.

Definition 5.1(a). We say that a pair of monoids $\left(C, C^{\prime}\right)$ is properly $c$-filtered whenever its filtration $\cdots \subseteq C_{d-1} \subseteq C_{d} \subseteq C_{d+1} \subseteq \cdots$ has the property that the inclusion ${ }^{\mathrm{id}}(C \times C)_{d} \subseteq(C \times C)_{d}$ is a cofibration.

(b) The pair $\left(C, C^{\prime}\right)$ is free whenever $C$ is a free monoid and $C^{\prime}$ is freely generated by a subset of the generators of $C$.

Theorem 5.2. Given a properly c-filtered free pair of monoids $\left(C, C^{\prime}\right)$, there is a principal fibration $\widetilde{C^{\prime}} \rightarrow \widetilde{C} \rightarrow \widetilde{C} / \widetilde{C^{\prime}}$, where $\widetilde{C} / \widetilde{C}^{\prime}$ is the topological group quotient of the naive group completions of $C$ and $C^{\prime}$ respectively.

Remark 5.3. By functoriality of our constructions, there is a commutative diagram

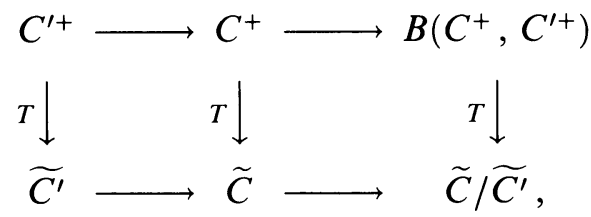

where, under the hypothesis of the theorem, the top row is a principal quasifibration (cf. [May75]) while the bottom row is a principal fibration. The induced maps on homotopy together with long exact sequences (for quasi- and principal fibrations) and the five lemma show that $T: \pi_{k}\left(B\left(C^{+}, C^{++}\right)\right) \rightarrow \pi_{k}\left(\widetilde{C} / \widetilde{C}^{\prime}\right)$ is an isomorphism.

Example 5.4. A. Long exact sequences for Lawson homology. Given a pair $\left(X, X^{\prime}\right)$ of projective algebraic sets, $X^{\prime}$ closed in $X$, we define the "relative Lawson homology" $L_{p} H_{n}\left(X, X^{\prime}\right)$ of the pair as the homotopy group

$$
\pi_{n-2 p}\left(B\left(\mathscr{C}_{p}(X)^{+}, \mathscr{C}_{p}\left(X^{\prime}\right)^{+}\right)\right) .
$$

It follows from the previous remark that one can use either the homotopy quotient $B\left(\mathscr{C}_{p}(X)^{+}, \mathscr{C}_{p}\left(X^{\prime}\right)^{+}\right)$or the naïve group quotient $\widetilde{\mathscr{C}}_{p}(X) / \widetilde{\mathscr{C}}_{p}\left(X^{\prime}\right)$ to define relative Lawson homology, and both cases yield the same long exact sequences for the theory.

B. Dold-Thom type results II. Essentially the same arguments used in the above example show that for a pair $\left(X, X^{\prime}\right)$ in the category $\mathscr{W}_{0}$ of finite CWcomplexes, the sequence $\widetilde{S P}\left(X^{\prime}\right) \rightarrow \widetilde{S P}(X) \rightarrow \widetilde{S P}(X) / \widetilde{S P}\left(X^{\prime}\right)$ is a principal fibration. Consequently one has a long exact sequence in homotopy:

$$
\cdots \pi_{k}\left(\widetilde{S P}\left(X^{\prime}\right)\right) \rightarrow \pi_{k}(\widetilde{S P}(X)) \rightarrow \pi_{k}\left(\widetilde{S P}(X) / \widetilde{S P}\left(X^{\prime}\right)\right) \rightarrow \pi_{k-1}\left(\widetilde{S P}\left(X^{\prime}\right)\right) \cdots
$$


At this stage, just the excision axiom remains to be proven for the functors $X \mapsto \pi_{k}(\widetilde{S P}(X))$ in the category $\mathscr{W}_{0}$. See Remark 5.6.

Lemma 5.5. Let $\left(C, C^{\prime}\right)$ be a pair of monoids satisfying the hypothesis of the Theorem 5.2. Then $\widetilde{C^{\prime}}$ is a closed subgroup of $\widetilde{C}$, and hence $\widetilde{C} / \widetilde{C}^{\prime}$ is a compactly generated abelian topological group. Furthermore, the quotient $\widetilde{C} / \widetilde{C^{\prime}}$ has a filtration by cofibrations given by

$$
Q_{d} \stackrel{\text { def }}{=} \pi\left(\widetilde{C}_{d}\right)=\pi \circ p\left((C \times C)_{d}\right),
$$

where $\widetilde{C}_{d}$ and $(C \times C)_{d}$ are the filtrations of $\widetilde{C}$ and $C \times C$ given respectively by Proposition 2.7 and Remark 2.5 .

Proof. We show that

$$
\operatorname{Sat}_{R}\left(C^{\prime} \times C^{\prime}\right)=\Delta_{C^{\prime}} \stackrel{\text { def }}{=} \Delta+\left(C^{\prime} \times C^{\prime}\right)
$$

as follows. If $p\left(m_{1}, m_{2}\right)=p\left(n_{1}, n_{2}\right)$ with $\left(n_{1}, n_{2}\right) \in C^{\prime} \times C^{\prime}$, we may assume that $n_{1}$ and $n_{2}$ have no common factors in their expression as sums of generators, otherwise if $\left(n_{1}, n_{2}\right)=\left(n_{1}^{\prime}+\lambda, n_{2}^{\prime}+\lambda\right)$ the freeness assumption implies that $\left(n_{1}^{\prime}, n_{2}^{\prime}\right) \in C^{\prime} \times C^{\prime}$, and hence $p\left(m_{1}, m_{2}\right)=p\left(n_{1}^{\prime}, n_{2}^{\prime}\right)$. Now, this is equivalent to $m_{1}+n_{2}=m_{2}+n_{1}$, and the previous argument shows that $m_{1}=n_{1}+\mu$ and $m_{2}=n_{2}+\mu$ for some $\mu \in C$. From Example B we conclude that $\operatorname{Sat}_{R}\left(C^{\prime} \times C^{\prime}\right)$ is closed in $C \times C$, and hence that $\widetilde{C^{\prime}}$ is a closed subgroup of $C$.

In order to show that $Q_{d-1} \subseteq Q_{d}$ is a cofibration we just need to observe that

$$
p:(C \times C)_{d} \backslash^{d}(C \times C)_{\mathrm{id}} \rightarrow Q_{d} \backslash Q_{d-1}
$$

is a bijection and to use Example $B$ and [Ste67, Lemma 8.4].

Proof of Theorem 5.2. It suffices to show that $\pi: \widetilde{C} \rightarrow \widetilde{C} / \widetilde{C^{\prime}}$ has a local cross section in a neighborhood of the identity $\langle\underline{0}\rangle \in \widetilde{C} / \widetilde{C^{\prime}}$, cf. [Ste51]. Let us start, inductively, requiring that the section takes the identity $\langle\underline{0}\rangle \in \widetilde{C} / \widetilde{C^{\prime}}$ to the identity $\underline{0} \in \widetilde{C}$, i.e., it takes $Q_{0}$ to $\widetilde{C}_{0}$.

Suppose, by induction, that we have constructed a section $\sigma_{k}: U_{k} \rightarrow \widetilde{C}$, where $U_{k}$ is open in $Q_{k} \subset \widetilde{C} / \widetilde{C^{\prime}}$.

From Proposition 4.7, we know that

$$
\pi \circ p:\left((C \times C)_{k+1},{ }^{\text {id }}(C \times C)_{k+1}\right) \rightarrow\left(Q_{k+1}, Q_{k}\right)
$$

is a relative homeomorphism of cofibrations. Let

$$
u_{k+1}:(C \times C)_{k+1} \rightarrow I, \quad h_{k+1}: I \times(C \times C)_{k+1} \rightarrow(C \times C)_{k+1}
$$

be a representation of $\left((C \times C)_{k+1},{ }^{\text {id }}(C \times C)_{k+1}\right)$ as a cofibration, and let $\widetilde{u_{k+1}}: Q_{k+1} \rightarrow I, \widetilde{h_{k+1}}: I \times Q_{k+1} \rightarrow Q_{k+1}$ be the corresponding representation for $\left(Q_{k+1}, Q_{k}\right)$ via $\pi \circ p$, as in Lemma 8.4 of [Ste67].

Define the open subsets

$$
W_{k+1} \stackrel{\text { def }}{=} u_{k+1}^{-1}([0,1)) \subset(C \times C)_{k+1}
$$

and

$$
\widetilde{W}_{k+1} \stackrel{\text { def }}{=}\left(\tilde{u}_{k+1}\right)^{-1}([0,1)) \subset Q_{k+1},
$$


and let

$$
\rho_{k+1}: W_{k+1} \rightarrow^{\text {id }}(C \times C)_{k+1}, \quad \tilde{\rho}_{k+1}: \widetilde{W}_{k+1} \rightarrow Q_{k}
$$

be the canonical retractions

$$
\left.\rho_{k+1} \stackrel{\text { def }}{=} h_{k+1}\right|_{\{1\} \times W_{k+1}},\left.\quad \tilde{\rho}_{k+1} \stackrel{\text { def }}{=} \tilde{h}_{k+1}\right|_{\{1\} \times \widetilde{W}_{k+1}},
$$

respectively.

Observe that $W_{k+1}=(\pi \circ p)^{-1}\left(\widetilde{W}_{k+1}\right)$ and that

$$
\left.\tilde{\rho}_{k+1} \circ(\pi \circ p)\right|_{W_{k+1}}=\left.(\pi \circ p)\right|_{\text {id }(C \times C)_{k+1}} \circ \rho_{k+1},
$$

by Lemma 8.4 of [Ste67].

Define $V_{k+1} \subset$ id $(C \times C)_{k+1}$ and $O_{k+1} \subset(C \times C)_{k+1}$ as the open subsets

$$
V_{k+1} \stackrel{\text { def }}{=}(\pi \circ p)^{-1}\left(U_{k}\right) \cap \text { id }(C \times C)_{k+1}
$$

and

$$
O_{k+1} \stackrel{\text { def }}{=} \rho_{k+1}^{-1}\left(V_{k+1}\right) \subset W_{k+1},
$$

where $U_{k} \subset Q_{k}$ is the domain of definition of the section $\sigma_{k}$. In particular $\rho_{k+1}$ restricts to a retraction $\rho_{k+1}: O_{k+1} \rightarrow V_{k+1}$.

The image

$$
U_{k+1} \stackrel{\text { def }}{=} \pi \circ p\left(O_{k+1}\right) \subset Q_{k+1}
$$

of $O_{k+1}$ under the projection $\pi \circ p$ is the open subset $\left(\tilde{\rho}_{k+1}\right)^{-1}\left(U_{k}\right)$, for

$$
\begin{aligned}
(\pi \circ p)\left(O_{k+1}\right) & =(\pi \circ p)\left\{\rho_{k+1}^{-1}\left(\left.(\pi \circ p)^{-1}\right|_{\text {id }(C \times C)_{k+1}}\left(U_{k}\right)\right\}\right. \\
& =(\pi \circ p)\left\{\left.(\pi \circ p)^{-1}\right|_{W_{k+1}} \circ\left(\tilde{\rho}_{k+1}\right)^{-1}\left(U_{k}\right)\right\} \\
& =\left(\tilde{\rho}_{k+1}\right)^{-1}\left(U_{k}\right),
\end{aligned}
$$

where the second equality comes from (5.5.1).

Consider the map $s_{k+1}: O_{k+1} \rightarrow \widetilde{C}$ given by sending $\alpha \in O_{k+1}$ to

$$
-p\left(\rho_{k+1}(\alpha)\right)+\sigma_{k}\left(\pi \circ p\left(\rho_{k+1}(\alpha)\right)\right),
$$

where $\sigma_{k}: U_{k} \rightarrow \widetilde{C}$ is the section defined inductively. Since

$$
\pi \circ s_{k+1}(\alpha)=-\pi \circ p\left(\rho_{k+1}(\alpha)\right)+\pi \circ \sigma_{k}\left(\pi \circ p\left(\rho_{k+1}(\alpha)\right)\right)=\langle\underline{0}\rangle \in \widetilde{C} / \widetilde{C^{\prime}},
$$

one sees that $s_{k+1}$ actually maps into $\widetilde{C^{\prime}}$.

Finally define $\sigma_{k+1}: U_{k+1} \rightarrow \widetilde{C}$ by $\sigma_{k+1}(u)=p(\alpha)+s_{k+1}(\alpha)$ where $\alpha \in$ $O_{k+1} \subset(C \times C)_{k+1}$ is representative of $u$, i.e., $\pi \circ p(\alpha)=u$. By definition, if $\alpha, \beta \in O_{k+1} \subset(C \times C)_{k+1}$ and $\alpha \sim \beta$ (i.e., $\pi \circ p(\alpha)=\pi \circ p(\beta)$ ) then either $\alpha=\beta$ or both $\alpha$ and $\beta$ lie in id $(C \times C)_{k+1} \cap O_{k+1}=V_{k+1}$. In the latter case $\rho_{k+1}(\alpha)=\alpha$ and $\rho_{k+1}(\beta)=\beta$, and hence

$$
\begin{aligned}
p(\alpha)+s_{k+1}(\alpha) & =p(\alpha)-p\left(\rho_{k+1}(\alpha)\right)+\sigma_{k}\left(\pi \circ p\left(\rho_{k+1}(\alpha)\right)\right) \\
& =\sigma_{k}(\pi \circ p(\alpha))=\sigma_{k}(\pi \circ p(\beta))=p(\beta)+s_{k+1}(\beta) .
\end{aligned}
$$

Therefore, the above identities show that $\sigma_{k+1}$ is well defined, $\sigma_{k+1} \circ(\pi \circ p)=$ $p+s_{k+1}$, and hence $\sigma_{k+1}$ is continuous (for $\pi \circ p$ is a proclusion), and also that

$$
\left.\sigma_{k+1}\right|_{U_{k+1} \cap(\pi \circ p)\left(V_{k+1}\right)}=\left.\sigma_{k+1}\right|_{U_{k}}=\sigma_{k}
$$


Noticing that

$$
\pi \circ \sigma_{k+1}(\pi \circ p(\alpha))=\pi\left(p(\alpha)+s_{k+1}(\alpha)\right)=\pi \circ j(\alpha)+\pi\left(s_{k+1}(\alpha)\right)=\pi \circ p(\alpha)
$$

one is able to construct, inductively a local cross section by defining $U=$ $\bigcup_{k} U_{k} \subset \widetilde{C} / \widetilde{C^{\prime}}$ and $\sigma: U \rightarrow \widetilde{C}$, with $\left.\sigma\right|_{U_{k}}=\sigma_{k}$. Since $U \cap Q_{k}=U_{k}$ is open in $Q_{k}$, the theorem is proven.

Concluding Remarks 5.6. In a similar fashion to the case of the homotopy groups of CW-complexes (Dold-Thom theorem [DT56]), Lawson homology also admits excision for pairs of projective varieties. See [LF91a] for a proof of this fact using naïve group completions and for applications to algebraic geometry.

The same type of arguments used in $\S 4$ and in [LF91a] yields excision-type results for pairs of monoids satisfying the conditions of $\S 5$. This would complete the Eilenberg-Steenrod axioms for the case of symmetric products.

Excision can also be obtained using the triple bar construction directly, supressing the use of naïve completions totally. This was actually written in earlier versions of this paper, but the relevance of such proof to the present work did not justify publishing it. A possible application for that, however, may turn to be in the search of localizations for the Lawson homology of varieties defined over arbitrary algebraically closed fields, as defined in [Fri91].

\section{REFERENCES}

[DL59] A. Dold and R. Lashof, Principal fibrations and fibre homotopy equivalence of bundles, Illinois J. Math. 3 (1959), 283-305.

[DT56] A. Dold and R. Thom, Quasifaserungen und unendliche symmetrische produkte, Ann. of Math. (2) 67 (1956), 230-281.

[Fri91] E. Friedlander, Algebraic cycles, Chow varieties and Lawson homology, Compositio Math. 77 (1991), 55-93.

[FL] E. Friedlander and H. B. Lawson, Jr., A theory of algebraic cocycles, Ann. of Math. (to appear).

[FM91] E. Friedlander and B. Mazur, Filtrations on the homology of algebraic varieties, preprint, 1991.

[Ful84] W. Fulton, Intersection theory, Springer-Verlag, Heidelberg, 1984.

[Hir75] H. Hironaka, Triangulation of algebraic sets, Proc. Sympos. Pure Math., vol. 29, Amer. Math. Soc., Providence, R.I., 1975, pp. 165-185.

[Hoy66] W. Hoyt, On the Chow bunches of different projective embeddings of a projective variety, Amer. J. Math. 88 (1966), 273-278.

[Law89] H. B. Lawson, Jr., Algebraic cycles and homotopy theory, Ann. of Math. (2) 129 (1989), 253-291.

[LF89] P. C. Lima-Filho, Homotopy groups of cycle spaces, Ph.D. thesis, SUNY, Stony Brook, N.Y., 1989.

[LF91a] _ Lawson homology for quasiprojective varieties, Compositio Math. 77 (1991).

[LF91b] _., On the generalized cycle map, Chicago, 1991.

[May72] J. P. May, The geometry of iterated loop spaces, Lecture Notes in Math., vol. 271, SpringerVerlag, Berlin and New York, 1972.

[May75] __, Classifying spaces and fibrations, Mem. Amer. Math. Soc. 155 (1975).

[Mil57] J. W. Milnor, The geometric realization of a semi-simplicial complex, Ann. of Math. (2) 65 (1957), 357-362.

[Mil67] J. Milgram, The bar construction and abelian h-spaces, Illinois J. Math. 11 (1967), 242-250. 
[MS76] D. McDuff and G. Segal, Homology fibrations and the group completion theorem, Invent. Math. 31 (1976), 279-284.

[Qui] D. Quillen, The group completion of a topological monoid, unpublished.

[Qui73] _, Higher algebraic K-theory, Lecture Notes in Math., vol. 341, Springer-Verlag, New York, 1973, pp. 85-147.

[Sam55] P. Samuel, Methodes d'algebre abstrait en geometrie algebrique, Springer-Verlag, Heidelberg and New York, 1955.

[Seg74] G. Segal, Categories and cohomology theories, Topology 13 (1974), 193-312.

[Sha74] I. Shafarevich, Basic algebraic geometry, Springer-Verlag, New York, 1974.

[Ste51] N. Steenrod, The topology of fiber bundles, Princeton Univ. Press, Princeton, N.J., 1951.

[Ste67] _ A convenient category of topological spaces, Michigan Math. J. 14 (1967), 133-152.

[Whi78] G. W. Whitehead, Elements of homotopy theory, Graduate Texts in Math., vol. 61, SpringerVerlag, New York, 1978.

Department of Mathematics, University of Chicago, 5734 University Avenue, Chicago, ILLINOIS 60637

Current address: Department of Mathematics, Texas A \& M University, College Station, Texas 77843 